${ }^{2}$ J. Lambe and R. C. Jaklevic, Phys. Rev. 165, 821 (1968).

${ }^{3}$ R. C. Jaklevic and J. Lambe, Bull. Am. Phys. Soc. 14, 43 (1969); J. Klein and A. Léger, Phys. Letters 28A, 134 (1968); J. M. Rowell, W. L. McMillan, and W. L. Feldmann, Phys. Rev. 180, 658 (1969); P. Guétin and G. Schréder, Solid State Commun. 8,291 (1970).

${ }^{4} \mathrm{~J}$. G. Adler, Phys. Letters 29A, 675 (1969).

${ }^{5}$ I. Giaever and H. R. Zeller, Phys. Rev. Letters $\underline{21}$, 1385 (1968).

${ }^{6}$ J. G. Adler, Solid State Commun. 7, 1635 (1969).

${ }^{7}$ R. C. Jaklevic and J. Lambe, Phys. Rev. B 2, 808 (1970).

${ }^{8}$ D. J. Scalapino and S. M. Marcus, Phys. Rev. Letters 18, 459 (1967).

${ }^{9}$ There may appear to be some ambiguity in the 16$\mathrm{meV}$ peak because the Au longitudinal-phonon energy is also in this range. However, a study of $\mathrm{Al}-\mathrm{Au}$ junctions indicates that the $\mathrm{Au}$ phonon structure is much weaker than what is observed here.

${ }^{10} \mathrm{C}$. Caroli, R. Combescot, P. Nozières, and D. Saint-James, J. Phys. C $\underline{5}, 21$ (1972).

${ }^{11} \mathrm{~J}$. Young and J. Koppel, Phys. Rev. 134, A1476 (1964).

${ }^{12} \mathrm{~A}$. Léger and J. Klein, Phys. Letters $28 \mathrm{~A}, 751$ (1969).

${ }^{13}$ It should be noticed that the electronic mean free path is always larger than the thickness of metal (1). In the case of $\mathrm{Pb}$, for electrons whose energy is $E \sim \theta_{\mathrm{Pb}}, \mathrm{Mc}-$ Millan and Rowell evaluate the value to be $500 \AA$ [Superconductivity, edited by R. D. Parks (Marcel Dekker, New York, 1969), p. 581]. This points out the difference between the electronic mean free path and the range of the inelastic-tunneling probe.

${ }^{14}$ M. J. L. Sangster, G. Pekham, and D. H. Saunderson, J. Phys. C 3 , 1026 (1970).

${ }^{15} \mathrm{C}$. B. Duke, Tunneling in Solids (Academic, New
York, 1969), p. 207.

${ }^{16}$ See, for instance, J. R. Schrieffer, Theory of Superconductivity (Benjamin, New York, 1964), Chap. IV; C. G. Kuper and G. D. Whitfield, Polarons and Excitons (Oliver and Boyd, Edinburgh, 1962).

${ }^{17}$ F. Ladan and A. Zylbersztejn, Phys. Rev. Letters 28, 1198 (1972).

${ }^{18}$ A. J. Bennett, C. B. Duke, and S. D. Silverstein, Phys. Rev. 176, 969 (1968).

${ }^{19}$ A. L. Geiger, B. S. Chandrasekhar, and J. G. Adler, Phys. Rev. 188, 1130 (1969).

${ }^{20}$ The $\mathrm{O}^{18}$ isotope was supplied by the Weizmann Institute (Rehovot).

${ }^{21}$ This value corresponds to the square root of the reduced-mass ratio as it is expected for optical phonons of zone center.

${ }^{22} \mathrm{G}$. Herzberg, Infra-red and Raman Spectra of Polyatomic Molecules (Van Nostrand, New York, 1945), p. 321.

${ }^{23}$ J. Gupta, Indian J. Phys. 10, 118 (1936).

${ }^{24} \mathrm{~L}$. J. Bellamy, in The Infra-red Spectra of Complex Molecules (Methuen, London, 1966).

${ }^{25} \mathrm{G}$. Herzberg, Spectra of Diatomic Molecules (Van Nostrand, New York, 1950), p. 560; N. Acquista, L. Schoen, and D. Lide, J. Chem. Phys. 48, 1534 (1968).

${ }^{26}$ There is a difference between the isotopic effect for $\mathrm{OH}-\mathrm{OD}$ free molecules and the square root of the reduced-mass ratio, because the vibrators a re anharmonic.

${ }^{27}$ R. A. Buchanan, H. H. Caspers, and J. Murphy, Appl. Optics 2, 1147 (1963).

${ }^{28}$ A. M. Glass and T. M. Searle, J. Chem. Phys. $\underline{46}$, 2092 (1967).

${ }^{29}$ S. S. Mitra, in Solid State Physics, edited by F. Seitz and D. Turnbull (Academic, New York, 1962), Vol. 13 , p. 1 .

${ }^{30} \mathrm{C} . \mathrm{B}$, Duke and G. G. Kleiman, Phys. Rev. B 2 , $1270(1970)$.

\title{
Optical Absorption in the Alkali Metals: Detailed Calculations
}

\author{
D. J. Stevenson ${ }^{*}$ \\ Victoria University of Wellington, New Zealand \\ (Received 10 August 1972)
}

\begin{abstract}
The results of detailed computations of the optical absorption in alkali metals are presented. Nettel's formalism is used to calculate the phonon-assisted processes and modifications are made to allow approximately for the Debye-Waller factor, multiphonon terms, anharmonic effects, and the "optical pseudopotential." The direct interband absorption is also incorporated, and the validity of the classical $\omega^{-2}$ frequency dependence for the Drude absorption is discussed. Results are presented as a function of temperature, form factor, and pseudopotential coefficient $V_{110}$. Smith's data for absorption in sodium are found to be consistent with $V_{110}\left(0^{\circ} \mathrm{K}\right)$ $=0.28 \mathrm{eV}$. The data for potassium and rubidium are also consistent with the model but the data for cesium and lithium are not.
\end{abstract}

\section{INTRODUCTION}

In recent years, there have been many experimental and theoretical investigations of the optical properties of the alkali metals. Despite this intensive activity, quantitative disagreement between experiment and theory remains. The objective of the present work is to examine whether the discrepancies can be explained without abandoning the single-particle formalism. In particular, the effect of phonons on the absorption is considered quantitatively. Recent papers ${ }^{1-3}$ have emphasized 
the importance of the phonons.

The simplest theoretical model for the real part of the conductivity $\sigma(\omega)$ leads to two principal contributions: (i) the Drude absorption with its characteristic $\omega^{-2}$ frequency dependence in the optical region and (ii) the direct interband absorption. Since considerable success has been achieved in describing the static conductivity of metals by the electron-phonon interaction, ${ }^{4}$ we might expect much of the Drude absorption to have a similar origin. Accordingly, we have calculated the total absorption as the sum of phonon-assisted absorption and direct interband absorption. We have found that the phonon-assisted absorption includes not only the Drude absorption but an indirect-interband contribution which substantially augments the Drude and direct-interband contributions.

In Sec. II we outline the formalism used. Nettel's method ${ }^{5}$ is used to calculate the phonon-assisted absorption, and the method of Ashcroft and Sturm $^{6}$ is used to calculate the direct-interband absorption. All relevant matrix elements are evaluated in the pseudopotential approximation in which the wave functions are expressed as the sum of two plane waves. In Sec. III the frequency dependence of the Drude absorption is critically examined and criteria are established for the validity of the often assumed $\omega^{-2}$ frequency dependence. In Sec. IV we examine the modifications required if due allowance is made for the thermal excitations of the crystal when matrix elements of the crystal potential are calculated. We find that there is excellent numerical cancellation of the two effects on the Drude absorption resulting from the Debye-Waller factor and the multiphonon terms. The Debye-Waller factor is still important in the interband absorption, however. In Sec. $V$ we show that anharmonic effects cannot be neglected in the alkali metals. In Sec. VI we present detailed results with emphasis on sodium and potassium. Changes in temperature, form factor, and pseudopotential coefficient $V_{110}$ are all considered. Finally, in Sec. VII we present general conclusions and discuss the inadequacies of past quantitative estimates.

\section{GENERAL FORMULATION}

We suppose that the total absorption can be written as the sum of a direct-interband contribution (which occurs even in the absence of phonons) and a phonon-assisted contribution. The latter is characterized by less stringent selection rules and by the absence of a threshold.

The phonon-assisted absorption can be calculated from second-order time-dependent perturbation theory. An equivalent approach has been developed by Nettel. ${ }^{5}$ The result obtained for the conductivity is

$$
\begin{aligned}
\sigma(\omega)= & \frac{\hbar}{6 \rho \omega(2 \pi)^{5}}\left(\frac{e}{m}\right)^{2} \sum \iint|\overrightarrow{\mathrm{M}}|^{2} f\left(\epsilon_{\overrightarrow{\mathrm{k}}-\overrightarrow{\mathrm{i}}, v}\right) \\
& \times\left[1-f\left(\epsilon_{\overrightarrow{\mathrm{k}}, u}\right)\right] \frac{2 n_{f t}+1}{\Omega_{f t}} \\
& \times \delta\left(\epsilon_{\overrightarrow{\mathrm{k}}, u}-\epsilon_{\overrightarrow{\mathrm{k}}-\overrightarrow{\mathrm{i}}, v}-\hbar \omega\right) d \overrightarrow{\mathrm{k}} d(\overrightarrow{\mathrm{k}}-\overrightarrow{\mathrm{f}})
\end{aligned}
$$

where

$$
\begin{aligned}
& \overrightarrow{\mathrm{M}}=S(u, v, \overrightarrow{\mathrm{k}}, \overrightarrow{\mathrm{f}}, t)[\overrightarrow{\mathrm{p}}(u, u, \overrightarrow{\mathrm{k}})-\overrightarrow{\mathrm{p}}(v, v, \overrightarrow{\mathrm{k}}-\overrightarrow{\mathrm{f}})] \\
& +S(w, v, \overrightarrow{\mathrm{k}}, \overrightarrow{\mathrm{f}}, t) \overrightarrow{\mathrm{p}}(u, w, \overrightarrow{\mathrm{k}}) \\
& -S(u, w, \overrightarrow{\mathrm{k}}, \overrightarrow{\mathrm{f}}, t) \overrightarrow{\mathrm{p}}(w, v, \overrightarrow{\mathrm{k}}-\overrightarrow{\mathrm{f}}), \\
& S(u, v, \overrightarrow{\mathrm{k}}, \overrightarrow{\mathrm{f}}, t)=\frac{N\langle\overrightarrow{\mathrm{k}}, u|\nabla \mu| \overrightarrow{\mathrm{k}}-\overrightarrow{\mathrm{f}}, v\rangle \cdot \overrightarrow{\mathrm{e}}_{f t}}{\epsilon_{\overrightarrow{\mathrm{k}}, \mu}-\epsilon_{\overrightarrow{\mathbf{k}}-\overrightarrow{\mathrm{f}}, v}},
\end{aligned}
$$

and

$$
\overrightarrow{\mathrm{p}}(u, v, \overrightarrow{\mathrm{k}})=\frac{\hbar}{i}\langle\overrightarrow{\mathrm{k}}, u|\vec{\nabla}| \overrightarrow{\mathrm{k}}, v\rangle \text {. }
$$

Here $\rho$ is the density of the crystal; $\vec{e}_{f t}$ is the polarization vector for a phonon with wave vector $\overrightarrow{\mathrm{f}}$, polarization $t$, and frequency $\Omega_{f t} ; N$ is the number of ions (unit volume is used throughout); and $\mu(\overrightarrow{\mathrm{r}})$ is the screened rigid-ion potential of an ion located at the origin. In Eq. (3), $u, v$, and $w$ label the final, initial, and intermediate states, respectively, while $n_{f t}$ and $f(\epsilon)$ are, respectively, the Bose-Einstein and Fermi-Dirac distribution functions. Finally, $\epsilon_{\overrightarrow{\mathrm{k}}, u}$ is the energy of an electron of wave vector $\overrightarrow{\mathrm{k}}$ in band $u$.

The formula for $\sigma(\omega)$ has the following limitations: (i) $\sigma(\omega)$ diverges at low frequencies because the electron-ion interaction is included to first order only. Pethick has shown ${ }^{7}$ that this approach is valid for $\omega \gg \Omega$; where $\Omega$ is a typical phonon frequency. This criterion is satisfied in the optical region. (ii) The harmonic approximation is made. The alkali metals are markedly anharmonic at room temperatures and in Sec. V some attempt is made to allow for this. (iii) Dynamic rather than static screening should be used. $\mathrm{Nettel}^{8}$ has discussed this possibility but Animalu finds only small corrections ${ }^{9}$ at the frequencies of interest.

We define the Drude absorption as that arising from the first two terms in the combined matrix element $\vec{M}$, since these terms do not involve interband dipole matrix elements. Our detailed results support this definition. The last two terms in $\vec{M}$ describe the indirect-interband absorption. These terms vanish if free-electron wave functions are used. There will also be interference between the contributions because the matrix element $\vec{M}$ appears squared in (1). The interference 
terms cannot be neglected in general. (Nettel's justification ${ }^{5}$ for neglecting the interference terms does not hold if the band gap is a significant fraction of the Fermi energy $\epsilon_{F}$. Thus, the cancellation of interference terms is far from complete, even in sodium.)

To evaluate the matrix elements, we assume that the single-particle valence bands are described in a local-pseudopotential approximation. Near a Brillouin zone $(\mathrm{Bz})$ face the resulting secular equation is truncated to a $2 \times 2$ determinant. ${ }^{6}$ The results of this can be written

$$
\begin{aligned}
\epsilon_{\overrightarrow{\mathbf{k}}}= & \frac{\hbar^{2}}{4 m}\left\{\overrightarrow{\mathrm{k}}^{2}+(\overrightarrow{\mathrm{k}}-\overrightarrow{\mathrm{G}})^{2}\right. \\
& \left. \pm\left[\left(2 \overrightarrow{\mathrm{k}} \cdot \overrightarrow{\mathrm{G}}-\overrightarrow{\mathrm{G}}^{2}\right)^{2}+4\left(\frac{2 m V_{G}}{\hbar^{2}}\right)^{2}\right]^{1 / 2}\right\}, \\
\psi_{\overrightarrow{\mathbf{k}}, 1}= & \frac{1}{\left(1+\gamma^{2}\right)^{1 / 2}}\left(e^{i \overrightarrow{\mathrm{k}} \cdot \overrightarrow{\mathrm{r}}}-\gamma e^{i(\overrightarrow{\mathrm{k}}-\overrightarrow{\mathrm{G}}) \cdot \overrightarrow{\mathrm{r}}}\right), \\
\psi_{\overrightarrow{\mathbf{k}}, 2}= & \frac{1}{\left(1+\gamma^{2}\right)^{1 / 2}}\left(\gamma e^{i \overrightarrow{\mathrm{k}} \cdot \overrightarrow{\mathrm{r}}}+e^{i(\overrightarrow{\mathrm{k}}-\overrightarrow{\mathrm{G}}) \cdot \overrightarrow{\mathrm{r}}}\right),
\end{aligned}
$$

where

$$
\gamma=\frac{\hbar^{2} \overrightarrow{\mathrm{k}}^{2} / 2 m-\epsilon_{\overrightarrow{\mathrm{k}}, 1}}{V_{G}},
$$

$\overrightarrow{\mathrm{G}}$ is a reciprocal-lattice vector of the first Brillouin zone, $V_{G}$ is the "folded" Fourier component ${ }^{6}$ appropriate to $\overrightarrow{\mathrm{G}}$, and $\epsilon_{\overrightarrow{\mathrm{k}}, 1}$ is given by the negative square root in (5). $\psi_{\vec{k}, 1}$ and $\psi_{\vec{k}_{0}, 2}$ are the normalized pseudo-wave-functions for, respectively, the lower and upper bands and for a particular arbitrary choice of phase. We shall write $V_{G}$ as $V_{110}$ since the first $\mathrm{Bz}$ of a body-centered-cubic (bcc) crystal is bounded by twelve (110) faces. For the alkali metals having this crystal structure, the Fermi surface is completely enclosed within the first $\mathrm{Bz}$ so that in an absorption process, the initial state is in the lower band and the final state can be in the lower or upper band. The dipole matrix elements can be easily evaluated:

$$
\begin{aligned}
& \overrightarrow{\mathrm{p}}(1,1, \overrightarrow{\mathrm{k}})=\hbar \overrightarrow{\mathrm{k}}-\hbar \overrightarrow{\mathrm{G}} \frac{\gamma^{2}}{1+\gamma^{2}}, \\
& \overrightarrow{\mathrm{p}}(2,2, \overrightarrow{\mathrm{k}})=\hbar \overrightarrow{\mathrm{k}}-\frac{\hbar \overrightarrow{\mathrm{G}}}{1+\gamma^{2}},
\end{aligned}
$$

$$
\overrightarrow{\mathrm{p}}(1,2, \overrightarrow{\mathrm{k}})=\overrightarrow{\mathrm{p}}(2,1, \overrightarrow{\mathrm{k}})=\frac{\hbar \gamma \overrightarrow{\mathrm{G}}}{1+\gamma^{2}},
$$

for the reduced zone scheme. The intraband dipole matrix elements are only slightly modified from their free-electron values (except for states very near a zone face), but the interband dipole matrix element depends critically on deviations from free-electron behavior. Rough calculations (which support the more detailed calculations of Politzer, Miskovsky, and Cutler ${ }^{10}$ ) show that the inclusion of the effect of the (200) faces changes the interband dipole matrix element by an amount which, while not insignificant, can be neglected in view of other approximations made. We have, however, allowed for the core-orthogonalization terms which Animalu incorporates in his "optical pseudopotential." 9 Essentially, this means replacing $V_{110}$ by $V_{110}+\hbar \omega P$ in evaluating the interband dipole matrix elements only, where $P$ is the intrinsically positive core projection operator. In our calculations, we have used the values tabulated by Animalu for $\hbar \omega=0.74 \epsilon_{F}$. (Recently, these have been disputed. ${ }^{11}$ )

To evaluate the electron-phonon matrix element $S(u, v, \overrightarrow{\mathrm{k}}, \overrightarrow{\mathrm{f}}, t)$, we suppose that we can replace the rigid-ion potential by a pseudopotential and the wave functions by pseudo-wave-functions. This procedure has been justified by Sham ${ }^{12}$ for the case of small excitation energies but appears to be less well justified otherwise, ${ }^{13}$ again because of the core-orthogonalization terms. We have not made a detailed allowance for these but approximate calculations suggest a $15 \%$ enhancement to the phonon-assisted absorption in sodium at $2 \mathrm{eV}$. (Nettel gives a figure of $10 \% .{ }^{5}$ ) The contributions to the indirect-interband absorption arising from excitations via a higher intermediate band (not included in our two-band model) were also roughly estimated for sodium and potassium and found to enhance the absorption by about $5 \%$ at $\hbar \omega=\epsilon_{F}$.

If the pseudo-wave-function corresponding to wave vector $\vec{k}-\vec{f}$ is written

$$
\psi_{1, \overrightarrow{\mathrm{k}}-\overrightarrow{\mathrm{f}}}=\frac{1}{\left(1+\gamma^{\prime 2}\right)^{1 / 2}}\left(e^{i(\overrightarrow{\mathrm{k}}-\overrightarrow{\mathrm{p}}) \cdot \overrightarrow{\mathrm{r}}}-\gamma^{\prime} e^{i\left(\overrightarrow{\mathrm{k}}-\overrightarrow{\mathrm{f}}-\overrightarrow{\mathrm{G}}^{\prime}\right) \cdot \overrightarrow{\mathrm{r}}}\right),
$$

then as an example, ${ }^{12}$

$$
S(1,1, \overrightarrow{\mathrm{k}}, \overrightarrow{\mathrm{f}}, t)=\frac{\overrightarrow{\mathrm{e}_{f t}} \cdot\left[\overrightarrow{\mathrm{f}} v(\overrightarrow{\mathrm{f}})-\gamma^{\prime}\left(\overrightarrow{\mathrm{f}}+\overrightarrow{\mathrm{G}}^{\prime}\right) v\left(\overrightarrow{\mathrm{f}}+\overrightarrow{\mathrm{G}}^{\prime}\right)-\gamma(\overrightarrow{\mathrm{f}}-\overrightarrow{\mathrm{G}}) v(\overrightarrow{\mathrm{f}}-\overrightarrow{\mathrm{G}})+\gamma \gamma^{\prime}\left(\overrightarrow{\mathrm{f}}-\overrightarrow{\mathrm{G}}+\overrightarrow{\mathrm{G}}^{\prime}\right) v\left(\overrightarrow{\mathrm{f}}-\overrightarrow{\mathrm{G}}+\overrightarrow{\mathrm{G}}^{\prime}\right)\right]}{\left(1+\gamma^{2}\right)^{1 / 2}\left(1+\gamma^{\prime 2}\right)^{1 / 2}\left(\epsilon_{\overrightarrow{\mathrm{k}}, 1}-\epsilon_{\overrightarrow{\mathrm{k}}-\overrightarrow{\mathrm{f}}, 1}\right)}
$$

where $v(\overrightarrow{\mathrm{f}})$ is the Fourier transform of the pseudopotential

$$
v(\overrightarrow{\mathrm{f}})=N \int v(\overrightarrow{\mathrm{r}}) e^{-i \overrightarrow{\mathrm{f}}, \overrightarrow{\mathrm{r}}} d \overrightarrow{\mathrm{r}} .
$$

The often used free-electron approximation to (10) $\left(r=\gamma^{\prime}=0\right)$ is sometimes valid in the static limit (see Collins and Ziman for a detailed analysis ${ }^{14}$ ) but becomes less valid as $\hbar \omega$ increases. 
We will refer to $v(\overrightarrow{\mathrm{f}})$ as the form factor (although the initial and final wave vectors need not lie on the Fermi surface for optical excitations), since we have used a local pseudopotential. In our calculations we have used both the detailed model form factors derived by Heine, Animalu, and Abarenkov (HAA) ${ }^{15}$ and the simple model devised by Ashcroft. ${ }^{16}$ For $f>2 k_{F}$, the HAA form factors are tabulated for backscattering which is an advantage since phonon-assisted absorption tends to be dominated by backscattering. The Ashcroft model is not expected to be very accurate for $f \geq 2 k_{F}$.

The phonon frequencies are calculated using the two-parameter model of Sharma and Joshi. ${ }^{17}$ This model is used to find frequencies at nonsymmetry points in the $\mathrm{Bz}$ by interpolation. The model can be fitted to within $5 \%$ of the experimental dispersion curves ${ }^{18}$ for all three modes and all three main symmetry directions for each of the alkali metals by making a suitable choice of elastic parameters. This simple model does not reproduce some of the structure in the phonon density of states (due to higher symmetry points), but since the absorption essentially involves an integral over the phonon spectrum, this should not be a serious deficiency.

To the contribution from (1), we should add the direct-interband absorption. This has been calculated using the Ashcroft-Sturm method and can be written for the alkali metals as ${ }^{6}$

$$
\begin{aligned}
\sigma_{I B}(\omega)=\frac{3 \sigma_{G}}{\pi}\left(a_{0} G\right) & \frac{\hbar \omega}{\epsilon_{G}} \int_{z_{0}^{2}}^{z_{1}^{2}} \frac{d y\left(\sqrt{y}+z_{0}\right)\left(z_{1}-\sqrt{y}\right)}{y^{3 / 2}(y-1)^{1 / 2}} \\
& \times \frac{2 z b}{\left(y-z^{2}+b^{2}\right)^{2}+4 z^{2} b^{2}},
\end{aligned}
$$

where

$$
\begin{aligned}
& \sigma_{\mathrm{G}}=\frac{e^{2}}{a_{0} \hbar} \frac{1}{24 \pi}=5.48 \times 10^{14} \mathrm{sec}^{-1}, \\
& G=\frac{2 \sqrt{2 \pi}}{a}, \quad \epsilon_{G}=\frac{\hbar^{2} G^{2}}{2 m}, \\
& z=\frac{\hbar \omega}{2\left|V_{110}\right|}, \quad b=\frac{\hbar}{2\left|V_{110}\right| \tau}, \\
& z_{0}=\left[2\left(\epsilon_{G} \epsilon_{F}+V_{1.10}^{2}\right)^{1 / 2}-\epsilon_{G}\right] / 2\left|V_{110}\right|, \\
& z_{1}=\left[2\left(\epsilon_{G} \epsilon_{F}+V_{110}^{2}\right)^{1 / 2}+\epsilon_{G}\right] / 2\left|V_{110}\right|,
\end{aligned}
$$

$a$ is the lattice parameter, $a_{0}$ is the first Bohr radius, and $\tau$ is the phenomenological relaxation time. If $\tau$ is chosen to be of the order of an electron-phonon relaxation time (about $10^{-14} \mathrm{sec}$ ), then Eq. (12) does not differ significantly from the Butcher result. ${ }^{19}$ The electron-electron relaxation time may be one or two orders of magnitude smaller; however, in which case the direct-interband threshold is smoothed out. In either case, our results of Sec. VI are not markedly changed so the choice of $\tau$ is not important in the alkalimetal interband absorption.

\section{DRUDE ABSORPTION}

In this section we restrict ourselves to that part of Eq. (1) which involves intraband dipole matrix elements only. Our purpose is to make some qualitative estimates of how closely this contribution [which we call the Drude conductivity $\sigma_{D}(\omega)$ ] follows the commonly accepted $\omega^{-2}$ frequency dependence.

We define a frequency-dependent optical relaxation time by generalizing the usual Drude formula ${ }^{20}$

$$
\sigma_{D}(\omega)=\frac{N e^{2}}{m \omega^{2} \tau_{\text {opt }}(\omega)}, \quad \omega \tau_{\text {opt }}(\omega) \gg 1 .
$$

If the classical theory is valid, $\tau_{\text {opt }}(\omega)$ should be weakly frequency dependent. To determine the qualitative features of this relaxation time we make several approximations. First, we evaluate the matrix elements using free-electron wave functions:

$$
\begin{aligned}
S(u, v, \overrightarrow{\mathrm{k}}, \overrightarrow{\mathrm{f}}, t)[\overrightarrow{\mathrm{p}}(u, u, \overrightarrow{\mathrm{k}})-\overrightarrow{\mathrm{p}} & (v, v, \overrightarrow{\mathrm{k}}-\overrightarrow{\mathrm{f}})] \\
= & i \overrightarrow{\mathrm{e}}_{f t} \cdot \overrightarrow{\mathrm{f}} v(\overrightarrow{\mathrm{f}}) \hbar \overrightarrow{\mathrm{f}} / \hbar \omega,
\end{aligned}
$$

where $\vec{f}$ is found by using the extended zone scheme. Equations (1) and (13) then combine to give

$$
\begin{aligned}
\frac{1}{\tau_{\text {opt }}(\omega)}= & \frac{\hbar}{6 N \rho m \omega(2 \pi)^{5}} \sum_{i} \iint \overrightarrow{\mathrm{f}}^{2}\left|\overrightarrow{\mathrm{e}}_{f t} \cdot \overrightarrow{\mathrm{f}}\right|^{2} v^{2}(\overrightarrow{\mathrm{f}}) f\left(\epsilon_{\overrightarrow{\mathrm{k}}-\overrightarrow{\mathrm{f}}, v}\right) \\
& \times\left[1-f\left(\epsilon_{\overrightarrow{\mathrm{k}}, u}\right)\right] \frac{2 n_{f t}+1}{\Omega_{f t}} \\
& \quad \times \delta\left(\epsilon_{\overrightarrow{\mathrm{k}}, u}-\epsilon_{\overrightarrow{\mathrm{k}}-\overrightarrow{\mathrm{f}}, v}-\hbar \omega\right) d \overrightarrow{\mathrm{k}} d(\overrightarrow{\mathrm{k}}-\overrightarrow{\mathrm{f}})
\end{aligned}
$$

We then use a model devised by Haga and Aisaka ${ }^{21}$ in which a modified Debye model (incorporating umklapp) is used for the phonons and an average over the direction of $\vec{f}$ is made. Using these approximations,

$$
\begin{aligned}
\frac{1}{\tau_{\text {opt }}(\omega)} & =\frac{2 m}{3 \rho \hbar^{2}(2 \pi)^{4}} \\
& \times \frac{1}{\hbar \omega} \int_{\epsilon_{1}}^{\epsilon_{F}} d \epsilon \int_{f_{2}-f_{1}}^{f_{2}+f_{1}} f^{3} S(f) v^{2}(f) d f,
\end{aligned}
$$

where

$$
\begin{aligned}
f_{1} & =\left(2 m / \hbar^{2}\right)^{1 / 2} \sqrt{\epsilon}, & & f_{2}=\left(2 m / \hbar^{2}\right)^{1 / 2}(\epsilon+\hbar \omega)^{1 / 2}, \\
\epsilon_{1} & =\epsilon_{F}-\hbar \omega, & & \hbar \omega<\epsilon_{F} \\
& =0, & & \hbar \omega \geq \epsilon_{F},
\end{aligned}
$$

and 


$$
S(f)=f^{2} W(f),
$$

where

$$
W(f)=\left\langle\left(2 n_{f t}+1\right) / \Omega_{f t}\right\rangle_{\mathrm{av}}
$$

is evaluated in Ref. 21. This is closely analogous to the result obtained by Wilson and Rice in their discussion of the Drude absorption in liquid metals. ${ }^{22}$ In the region $1 / \tau_{\text {opt }(\omega)} \ll \omega \ll \epsilon_{F} / \hbar$, we can expand (16) in a power series

$$
\begin{aligned}
\frac{1}{\tau_{\text {opt }(\omega)}} & =\frac{1}{\tau_{\text {opt }(0)}} \\
& \times\left[1+\alpha_{1}\left(\frac{\hbar \omega}{\epsilon_{F}}\right)+\alpha_{2}\left(\frac{\hbar \omega}{\epsilon_{F}}\right)^{2}+\cdots\right]
\end{aligned}
$$

where

$$
\frac{1}{\tau_{\text {opt }}(0)}=\frac{2 m}{3 \rho \hbar^{2}(2 \pi)^{4}} \int_{0}^{2 k_{F}} f^{3} v^{2}(f) S(f) d f
$$

and $k_{F}$ is the free-electron Fermi wave vector. It can readily be shown that $\alpha_{1}$ is proportional to $v^{2}\left(2 k_{F}\right)$ and $\alpha_{2}$ is proportional to $v\left(2 k_{F}\right)$. This suggests that a criterion for the validity of the classical theory is that $v\left(2 k_{F}\right)$ should be small [compared, say, with the average value of $|v(f)|$ over the region of integration]. This is certainly satisfied by metals such as sodium and potassium but may not be satisfied by metals such as lithium and silver. Equation (16) has been evaluated for a number of metals for arbitrary choices of form factor. Some typical results are shown in Fig. 1 for sodium. The HAA form factor is found to give a weak frequency dependence. There may be a tendency for $1 / \tau_{\text {opt }}(\omega)$ to decrease for $\hbar \omega \gtrsim \epsilon_{F}$.

(a)

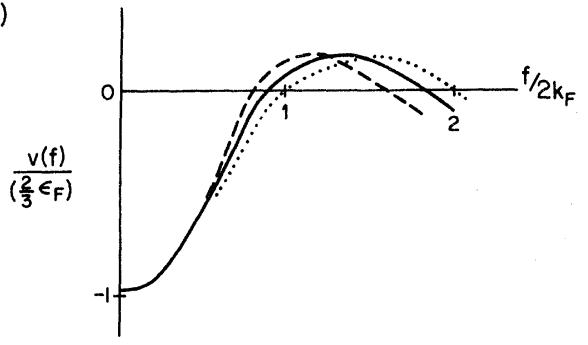

(b)

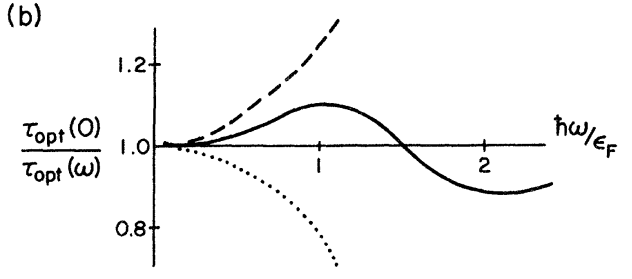

FIG. 1. Effect of various form factors on the form of the Drude absorption for sodium: solid line HAA form factor; dashed and dotted lines arbitrary modifications.
This is a "saturation" effect which results because all the conduction electrons can now be excited. [The range of integration for $\epsilon$ in (16) is no longer increasing and so the $1 / \hbar \omega$ preceding the integration sign is uncompensated.]

The calculations outlined above, although of limited applicability, demonstrate that the general formula for $\sigma_{D}(\omega)$ derived from (1) does not guarantee a $\omega^{-2}$ frequency dependence. This frequency dependence seems likely, however, in those cases where the electrons are weakly perturbed from free particles (e.g., sodium, potassium, aluminum). The more exact calculations of Sec. VI confirm this assertion.

One other related criterion for the valiaity of the classical Drude result is evident if we observe that except for angular factors, we may set in (15)

$$
\delta\left(\epsilon_{\overrightarrow{\mathbf{k}}, u}-\epsilon_{\overrightarrow{\mathrm{k}}-\overrightarrow{\mathrm{f}}, v}-\hbar \omega\right) \cdot \overrightarrow{\mathrm{k}} d(\overrightarrow{\mathrm{k}}-\overrightarrow{\mathrm{f}}) \sim D(\epsilon) D(\epsilon+\hbar \omega) d \epsilon,
$$

where $D(\epsilon)$ is the density of states at the initial energy $\epsilon$. If we could ignore the rest of the integrand in (15) we would have

$$
\frac{1}{\tau_{\mathrm{opt}}(\omega)} \sim \frac{1}{\hbar \omega} \int_{\epsilon_{1}}^{\epsilon_{F}} D(\epsilon) D(\epsilon+\hbar \omega) d \epsilon 。
$$

For the free-electron case $D(\epsilon) \sim \sqrt{\epsilon}$ and $1 / \tau_{\text {opt }}(\omega)$ is slowly varying for $\hbar \omega \lesssim \epsilon_{F}$. In a metal where $D(\epsilon)$ varies markedly over the region of integration we might expect $1 / \tau_{\text {opt }}(\omega)$ to be more strongly frequency dependent. This is probably not important in the alkali metals. We have, however, done a calculation for a non-alkali-metal, namely, silver, which suggests a $30 \%$ drop in $1 / \tau_{\text {opt }}(\omega)$ as $\hbar \omega$ increases from 1 to $4 \mathrm{eV}$. In the absence of other compensating effects, this should be experimentally observable. This conclusion is approximately independent of the approximations made since the rest of the integrand in (15) is slowly varying.

\section{DEBYE-WALLER FACTOR AND MULTIPHONON EFFECTS}

When calculating the absorption we should allow for the thermal excitations of the crystal. It is well known that these modify the intensity of a scattering process, calculated to lowest order, by $e^{-2 W(\overrightarrow{\mathrm{f}})}$, where $W(\overrightarrow{\mathrm{f}})$ is the Debye-Waller factor ${ }^{23}$

$$
W(\overrightarrow{\mathrm{f}})=\sum_{\overrightarrow{\mathrm{q}}, t} \frac{\hbar}{4 M N \Omega_{q t}}\left|\overrightarrow{\mathrm{f}} \cdot \overrightarrow{\mathrm{e}}_{q t}\right|^{2}\left(2 n_{q t}+1\right) .
$$

In (19), $M$ is the ion mass, $\overrightarrow{\mathrm{f}}$ is the wave-vector change in the scattering, and the sum is over the first Bz. As Sturm has shown, ${ }^{1}$ this means that the integrand in (12) should include an additonal factor of $e^{-2 W(\vec{G})}$. The phonon-assisted absorption is modified in a more complicated way, however.

In the Drude absorption we should replace $S(u, v, \overrightarrow{\mathrm{k}}, \overrightarrow{\mathrm{f}}, t)$ by $e^{-W(f)} S(u, v, \overrightarrow{\mathrm{k}}, \overrightarrow{\mathrm{f}}, t)$ while the intra- 
TABLE I. Sodium at $300^{\circ} \mathrm{K}$. Contributions to absorption as a percentage of one-phonon contribution.

\begin{tabular}{lccr}
\hline \hline $\begin{array}{l}\hbar_{\omega} \\
(\mathrm{eV})\end{array}$ & Debye-Waller & Two phonon & Total \\
\hline 0 & $-12 \%$ & $+17 \%$ & $+5 \%$ \\
1 & $-17 \%$ & $+18 \%$ & $+1 \%$ \\
2 & $-21 \%$ & $+23 \%$ & $+2 \%$ \\
\hline
\end{tabular}

band dipole matrix element is unmodified since it is evaluated between a state and itself. In addition, however, we should include multiphonon terms. Sham and Ziman ${ }^{23}$ show that these occur in first-order perturbation theory because the ion displacement must be expressed as a sum of lattice modes. They also show qualitatively that these terms tend to compensate for the reduction in the matrix element due to the Debye-Waller factor. We have carried out computer calculations to test the extent of this compensation, using the formulas derived by Sham and Ziman (p. 229 of Ref. 23). Contributions of order $T^{2}$ to the Drude absorption are calculated in the high-temperature limit (all $n_{f t} \gg 1$ ) for sodium. Similar results should hold for other metals. The results will depend on the form factor chosen: The HAA form factor ${ }^{15}$ is used here. Care was taken to allow for all two-phonon processes (there are 36 of them since each phonon can have one of three polarizations and can be either emitted or absorbed). To evaluate the integrals, a Monte Carlo method was used (see Sec. VI). Table I shows that the cancellation is excellent. (The figures quoted are accurate to about \pm 1 .) The results for $\hbar \omega=0$ can be interpreted as the contributions to the dc resistivity (which, like the Drude conductivity, varies as $1 / \tau$ in the classical theory) and are in good agreement with the constant density data of Dugdale and Gugan $^{24}$ which suggest about a $7 \%$ positive contribution at $300^{\circ} \mathrm{K}$ arising from a $T^{2}$ term in the resistivity.

In indirect-interband processes, both the electron-phonon and dipole matrix elements are modified by the Debye-Waller factor. The first of these modifications should be compensated for by the multiphonon indirect-interband processes and so to a first approximation, the indirect-interband contribution to $\sigma(\omega)$ scales as $e^{-2 W(\overrightarrow{\mathrm{G}})}$ - the same as the direct interband.

\section{ANHARMONIC EFFECTS}

It would be a difficult task to rederive $\mathrm{Eq}_{\text {. }}$ (1) rigorously allowing for anharmonic terms. This has not been done and is a limitation of our present analysis. We can, however, allow for two important anharmonic effects. These are the temperature dependences of the phonon spectrum and the band gap.

The results of Buyers and Cowley ${ }^{25}$ for potassium indicate about a $10 \%$ average drop in the phonon frequencies in going from 9 to $299^{\circ} \mathrm{K}$. To a good approximation, it is the square of the phonon frequency which enters into the calculations of phonon-assisted absorption. A similar change is expected in the other alkalis (as Martinson's results for sodium indicate ${ }^{26}$ ), with the probable exception of lithium which has a much higher melting point. An effect of this magnitude (i. e., 10\%) is allowed for in our detailed calculations of Sec. VI.

The change in the band gap $\left(=2 V_{110}\right.$ in our model), between absolute zero and room temperature, cause by lattice expansion may be substantial in the alkali metals. Ham's band-structure calculations ${ }^{27}$ indicate that the potassium band gap decreases by about $15 \%$ for this temperature change (even though the lattice parameter changes from 5.22 to $5.33 \AA$ only). The same change can be predicted by Ashcroft's empty-core pseudopotential ${ }^{16}$ by using a temperature-independent empty-core radius of $2.11 a_{0}$ and allowing for the change in $k_{F}$ with temperature. In Fig. 2 we plot an effective pseudopotential coefficient defined by

$$
V_{110}^{\text {eff }}(T)=e^{-W(\vec{G})} V_{110}(T),
$$

where $V_{110}(T)$ is the coefficient found using the Ashcroft model [for a particular choice of $V_{110}(0)$ ] and allowing for lattice expansion. About half the temperature dependence shown is caused by lattice expansion; the other half results from the Debye-Waller factor. Since the interband absorption varies as $\left[V_{110}^{\text {eff }}(T)\right]^{2}$ (if we neglect the $\hbar \omega P$ orthogonalization term), Fig. 2 shows that we should not try to fit room-temperature absorption using a pseudopotential coefficient found from lowtemperature de Haas-van Alphen (dHvA) results. Careful allowance must be made for temperature.

\section{DETAILED RESULTS}

The formulas given in Sec. II. are used to evaluate the total optical absorption for the alkali metals in the frequency range covered by Smith's ex-

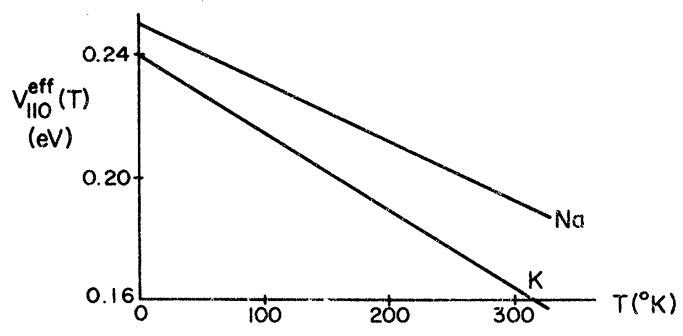

FIG. 2. Effective value of $V_{110}$ allowing for both lattice expansion and the Debye-Waller factor. 
periments. ${ }^{28,29}$ There is a problem in comparing theory with experiment since our calculation is for bulk absorption whereas there is increasing experimental evidence that the boundary conditions of the sample have an important effect on the results. $^{39}$ For example, our calculations for sodium are not compatible with the experimental results of Mayer and Hietel ${ }^{42}$ or the vacuum interface data of Palmer and Schnatterly。 ${ }^{39}$ It is not obvious why Smith's results should be preferred. The phonon-assisted absorption is calculated by a Monte Carlo technique. Initial and final wave vectors are generated using a random-number generator in this method, and a histogram of $\sigma(\omega)$ vs $\hbar \omega$ is built up. Convergence to within $5 \%$ is obtained eventually. As a test of our method, the Debye-Waller factors for the alkali metals have been accurately calculated using Eq. (19). Excellent agreement with the experimental data for sodi$\mathrm{um}^{30}$ is obtained. In the calculation of Eq. (1), allowance must be made for two singularities. The pole at $\overrightarrow{\mathrm{f}}=\overrightarrow{\mathrm{G}}$ (where the phonon frequency goes to zero) is integrable and can be allowed for by placing a small sphere around it and averaging over the volume enclosed. The pole arising from the vanishing of the energy denominator in $S(u, v, \overrightarrow{\mathrm{k}}, \overrightarrow{\mathrm{f}}, t)$ is not directly integrable but leads to a principal parts integral as discussed by Chester. ${ }^{31}$ The direct-interband absorption is easily calculated by conventional numerical integration. In our calculations we have made approximate allowance for the following effects as discussed in previous sections: the "optical pseudopotential," the DebyeWaller factor, multiphonon terms in first-order perturbation theory, and anharmonicities. We did not allow for electron-electron scattering (the effect of which is controversial ${ }^{32}$ ), surface or bulk plasmons ${ }^{33}$ higher pseudopotential coefficients $\left(V_{200}, V_{211}\right.$, etc. $)$, the anomalous skin effect, ${ }^{34}$ or higher-order phonon processes. As a consequence, we expect the results to be most accurate at the lowest frequencies. The Fermi level for each metal is fixed by integrating the density of states appropriate to Eq. (5) ${ }^{35}$ from the bottom of the conduction band up to an energy level such that the right number of conduction electrons is obtained. $\quad V_{110}$ is treated as a variable parameter although it should strictly lie on the form factor. The graphs are labeled by $V_{110}\left(0^{\circ} \mathrm{K}\right)$ but the calculations are made with the correct parameter $V_{110}^{e f f}(T)+\hbar \omega P$. Thus, the parameters labeling the graphs can be directly compared with the values of $V_{110}$ found to fit the low-temperature dHvA measurements.

\section{A. Sodium}

Figure 3 shows that the HAA form factor ${ }^{15}$ predicts the Drude absorption observed by $\mathrm{Smith}^{28}$ to within $10 \%$. (Although not shown on the graph, agreement extends back to the lowest frequencies for which Smith has results.) The Drude absorption closely follows the $\omega^{-2}$ dependence at the lowest frequencies but is supplemented at the higher frequencies by the indirect-interband absorption. At $2.4 \mathrm{eV}$ the contributions to absorption due to direct, Drude, and indirect interband are about equal. The interband region is best fitted by $V_{110}\left(0^{\circ} \mathrm{K}\right)=0.28$ or $-0.45 \mathrm{eV}$. The former is favored since it is close to the value of $0.25 \mathrm{eV}$ that is consistent with Lee's dHvA results. ${ }^{36}$ The $0.03-\mathrm{eV}$ discrepancy is not significant in view of the approximations made and the absorption processes not allowed for. The HAA form factor gives $V_{110}\left(0^{\circ} \mathrm{K}\right)=0.27 \mathrm{eV}$ so our calculation is consistent.

The calculation has been made only with the Animalu pseudopotential. ${ }^{9}$ Recent calculations by Bennett and Vosko ${ }^{11}$ suggest inadequacies in the

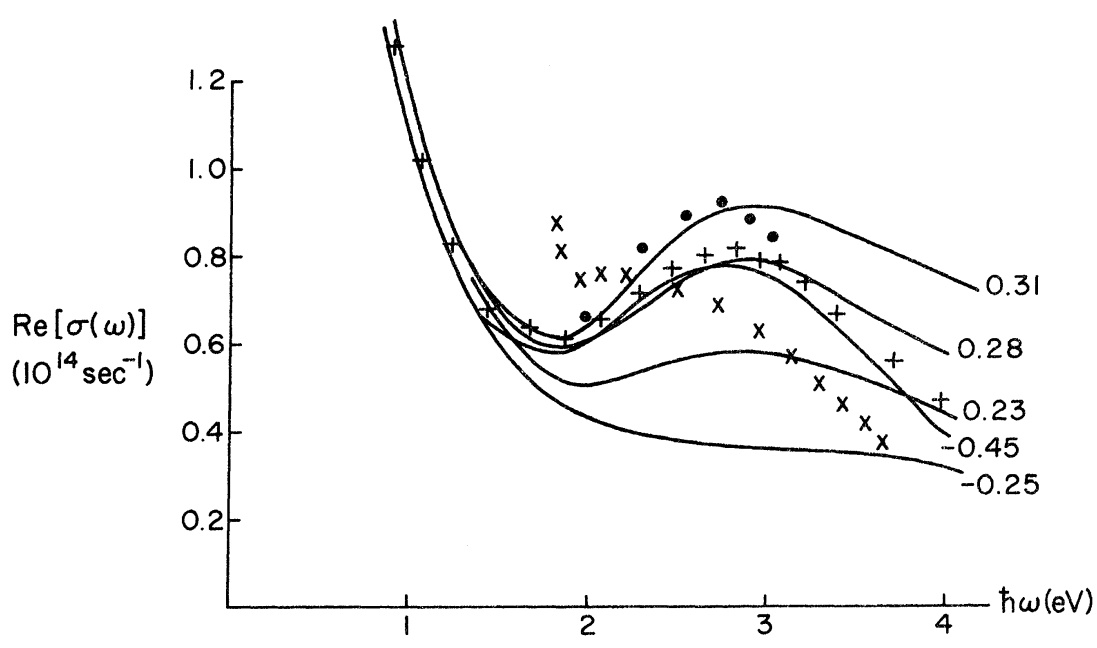

FIG. 3. Total absorption in sodium at room temperature: +, Smith (Ref. 28); $\times$, Palmer and Schnatterly (Ref. 39) (vacuum interface); $\bullet$, Palmer and Schnatterly (Ref. 39) (glass-sodium interface); solid line, theoretical results as a function of $V_{110}^{\text {eff }}(0)$ in eV. 


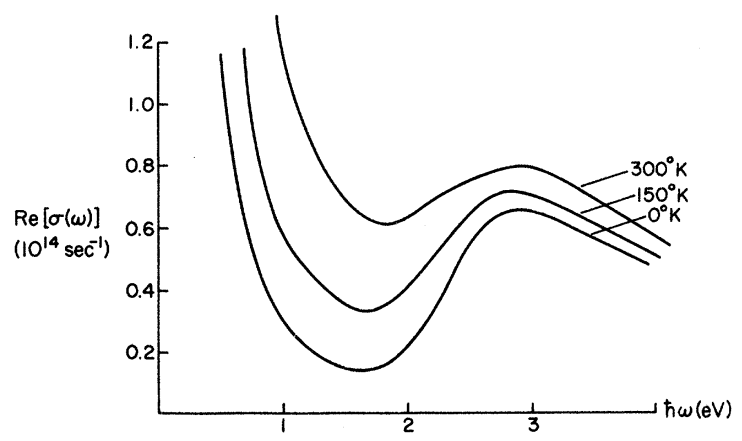

FIG. 4. Absorption in sodium as a function of temperature. Theoretical predictions, using $V_{110}^{\text {eff }}(0)=0.28$ eV.

details of Animalu's calculation but their final result does not differ very significantly. Bennett and Vosko obtain agreement with Smith's experimental results only by allowing for the substantial electron-electron contribution given by Gurzhi's theory. ${ }^{32}$ This is of questionable validity since Gurzhi's result is only likely to be valid for $\hbar \omega \ll \epsilon_{F}$. We estimate that use of the Bennett and Vosko optical pseudopotential gives $0.26 \mathrm{eV}$ for $V_{110}\left(0^{\circ} \mathrm{K}\right)$ instead of $0.28 \mathrm{eV}$.

For $V_{110}\left(0^{\circ} \mathrm{K}\right)=0.28 \mathrm{eV}$, the temperature dependence of $\sigma(\omega)$ has been calculated and the results are shown in Fig. 4. The Drude absorption varies linearly with temperature at high temperatures but does not go to zero at low temperatures because phonon emission is always possible for $\omega>\Omega$. The weak dependence on temperature of the high-frequency absorption is due to the competing effects of increasing $V_{110}^{\text {eff }}(T)$ and decreasing phonon population as the temperature is lowered.

An attempt has been made to fit the absorption using the Ashcroft pseudopotential ${ }^{16}$ but this is less satisfactory than the HAA model. An empty-core parameter of $R_{\theta}=1.84 a_{0}$ fits the Drude absorption but greatly overestimates $V_{110}\left(0^{\circ} \mathrm{K}\right)$. The value of $R_{0}$ which best fits the liquid-metal resistivity $\left(1.66 a_{0}\right)^{37}$ overestimates the Drude absorption by $40 \%$. This further emphasizes the importance of the choice of the form factor. ${ }^{38}$

\section{B. Potassium}

In Fig. 5 we see that the HAA form factor is also successful in predicting the Drude absorption in potassium. Once again, no significant deviations from $\omega^{-2}$ behavior are found at low frequencies. Reasonable agreement in the interband region is indicated for $V_{110}\left(0^{\circ} \mathrm{K}\right)=0.15$ or -0.25 $\mathrm{eV}$. The HAA form factor gives $0.12 \mathrm{eV}$. However, Ham's band-structure calculation ${ }^{27}$ suggests a value of $-0.23 \mathrm{eV}$. The discrepancy here is probably due to the nonlocal nature of the pseudopotential. Lee and Falicov ${ }^{39}$ found that the dHvA

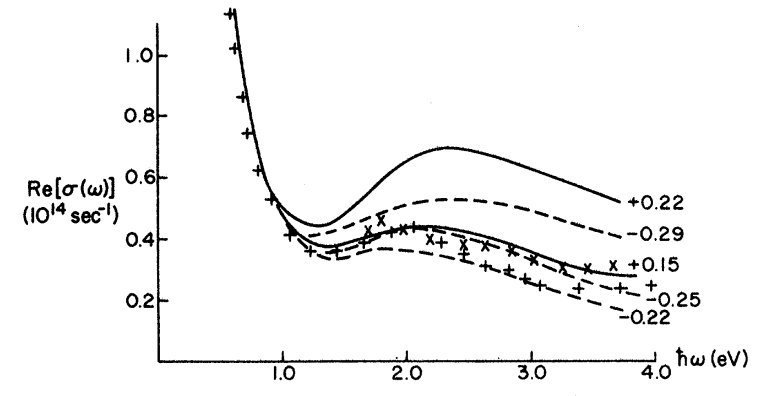

FIG. 5. Total absorption in potassium at room temperature: +, Smith (Ref. 28); $\times$, Palmer and Schnatterly (Ref. 39); dashed and solid lines, theoretical results as a function of $V_{110}^{\text {eff }}(0)$ in $\mathrm{eV}$.

results could only be explained by substantial nonlocal contributions.

For $V_{110}\left(0^{\circ} \mathrm{K}\right)=-0.25 \mathrm{eV}$, the temperature dependence of $\sigma(\omega)$ is shown in Fig. 6. The slight rise in absorption as the temperature is lowered may not be significant and becomes a slight drop if $V_{110}\left(0^{\circ} \mathrm{K}\right)=0.15 \mathrm{eV}$ is used instead. The results of Smith, ${ }^{28}$ Palmer and Schnatterly, ${ }^{40}$ and Yamaguchi and Hanyu ${ }^{41}$ all agree quite closely in the interband region and all disagree with Mayer and Hietel's results. ${ }^{42}$ Moreover, Palmer and Schnatterly found the high-frequency absorption in potassium to be very weakly temperature dependent. This suggests that the phonon-assisted absorption is indeed as important as we calculate it to be, since if direct-interband absorption predominated, a marked increase in absorption would be expected as the temperature is lowered.

\section{Rubidium}

In rubidium, the HAA form factor is found to overestimate the Drude absorption observed by Smith $^{29}$ and Mayer and von Aufschnaiter ${ }^{43}$ by about 100\%. A fit can be obtained using the Ashcroft model; however, for $R_{e}=2.09 a_{0}$ or $2.52 a_{0}$. The total absorption is shown in Fig. 7 for the choice $R_{e}=2.09 a_{0}$. Good agreement with Smith's results is possible for $V_{110}\left(0^{\circ} \mathrm{K}\right)=-0.21$ or $0.17 \mathrm{eV}$.

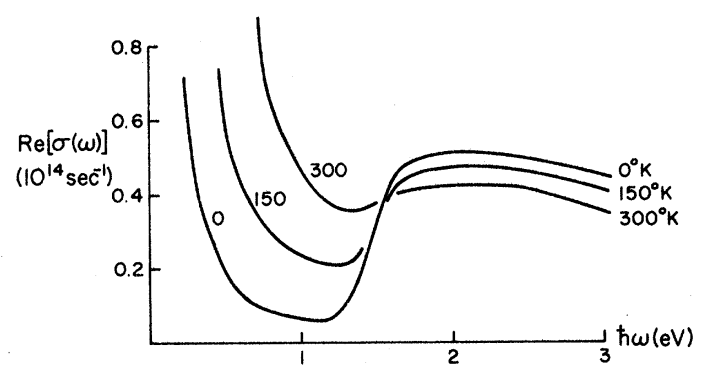

FIG. 6. Absorption in potassium as a function of temperature. Theoretical predictions for $V_{110}^{\text {eff }}(0)=-0.25 \mathrm{eV}$. 


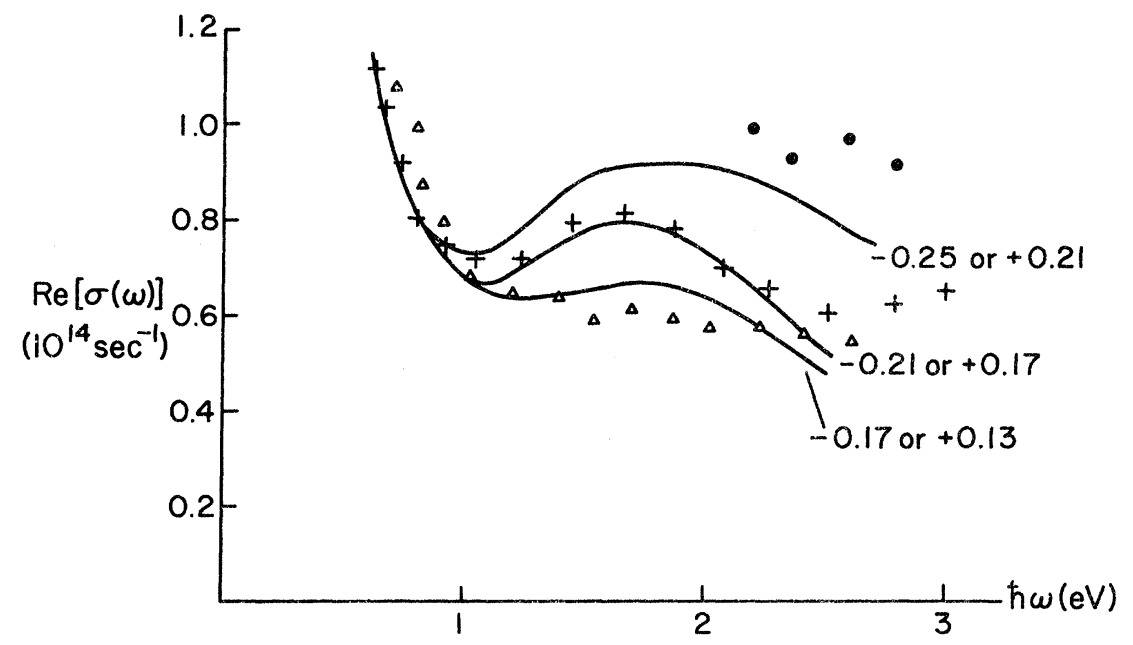

FIG. 7. Total absorption in rubidium at room temperature: + , Smith (Ref. 29); $\Delta$, Von Aufschnaiter (Ref. 43); $\bullet$, Ives and Briggs (Ref. 44); solid line, theoretical results as a function of $V_{110}^{\text {eff }}(0)$ in $\mathrm{eV}$.
These can be compared with the value $\left|V_{110}\right|=0.43$ $\mathrm{eV}$ which Ashcroft deduced ${ }^{45}$ from dHvA data, and Ham's band-structure calculation ${ }^{27}$ which gives $V_{110}=-0.37 \mathrm{eV}$. These values seem too large to be consistent with the optical absorption. However, the local-pseudopotential approach is likely to be invalid for rubidium. The temperature dependence of absorption in rubidium should be similar to that of sodium and potassium.

\section{Cesium}

As for rubidium, the HAA form factor for cesium is found to overestimate the Drude absorption observed by Smith ${ }^{29}$ by a factor of 2 (if we suppose the two lowest-frequency data points he has obtained are indicative of the Drude absorption). A fit is obtained with the Ashcroft model for $R_{e}=2.35 a_{0}$ or $2.50 a_{0}$. However, the very low threshold for interband absorption which Smith and Mayer and Hietel ${ }^{42}$ have observed is incompatible with our two-band model. Smith suggests that the low threshold has its origin in the excitation of conduction electrons to a low-lying $d$ band.

\section{E. Lithium}

Lithium is so unlike the other alkali metals as probably to require special treatment. Moreover, there is little agreement between the presently available results of Hodgson ${ }^{46}$ and those of Mathewson and Myers. ${ }^{47}$ We regard the results of Mathewson and Myers as more reasonable since their low-frequency data show a temperature de-. pendence consistent with Eq. (15). Hodgson's results appear to include a large temperature-independent contribution to the Drude absorption, the origin of which is not known. However, Mathewson. and Myers have observed an absorption minimum near $2 \mathrm{eV}$ whereas our two-band model predicts an absorption minimum at around $3 \mathrm{eV}$ for any reasonable choice of $V_{110}\left(0^{\circ} \mathrm{K}\right)$. This suggests that either the lithium band structure is quite unlike our two-band model (which is not supported by band-structure calculations ${ }^{27,48}$ ) or that the metal is in a different phase from bcc. At $78^{\circ} \mathrm{K}$ there is a partial martensitic transformation from bcc to the hexagonal close-packed modification. It is possible that a metallic film may remain metastable in that phase above $78^{\circ} \mathrm{K}$.

We have fitted the Ashcroft pseudopotential to the low-frequency results of Mathewson and Myers for $R_{e}=1.17 a_{0}$ and $1.84 a_{0}$. Our calculations predict that the Drude absorption has a significant positive deviation from $\omega^{-2}$ behavior $(15 \%$ at $2 \mathrm{eV})$ which is in qualitative agreement with their results.

\section{DISCUSSION}

The main results are listed in Table II. The good agreement between our calculated values of $V_{110}\left(0^{\circ} \mathrm{K}\right)$ and the values of $V_{110}$ consistent with dHvA data for sodium and potassium suggests that the single-particle formalism adequately describes Smith's experimental results for these two metals. More accurate calculations in these and other metals may require a nonlocal pseudopotential and a more accurate wave function. We conclude that past analyses are at fault at least in that they do not allow for the substantial temperature dependence of the pseudopotential coefficient. In particular, our calculation gives only half the indirect-interband absorption that Miskovsky and Cut$\operatorname{ler}^{2}$ have calculated in sodium. We find that we can restore agreement with experiment by adding the direct-interband absorption (which Miskovsky and Cutler do not appear to have done). Also, we did not get the dip they have calculated to occur at around $2.4 \mathrm{eV}$ in sodium, although we have also used a step function for the Fermi distribution. 
TABLE II. Main results.

\begin{tabular}{|c|c|c|c|c|c|}
\hline & $\begin{array}{c}\left|V_{110}\right| \\
\text { (dHvA } \\
\text { (Ref. 45) } \\
(\mathrm{eV})\end{array}$ & $\begin{array}{c}V_{110} \\
(\mathrm{Ham}, \mathrm{Ref} .27)\end{array}$ & $\begin{array}{c}V_{110}\left(0^{\circ} \mathrm{K}\right) \\
\text { (present } \\
\text { calculation) } \\
(\mathrm{eV})\end{array}$ & $\begin{array}{c}R_{e}\left(a_{0}\right) \\
\text { (liquid } \\
\text { resistivity, Ref. 27) }\end{array}$ & $\begin{array}{l}R_{e}\left(a_{0}\right) \\
\text { (present } \\
\text { calculation) }\end{array}$ \\
\hline $\mathrm{Li}$ & $\cdots$ & 1.37 & $\cdots$ & $1.06,2.00$ & $1.17,1.84$ \\
\hline $\mathrm{Na}$ & $0.25^{\mathrm{a}}$ & 0.11 & $0.28,-0.45$ & 1.67 & 1.84 \\
\hline $\mathrm{K}$ & $0.24^{\mathrm{b}}$ & -0.23 & $-0.25,0.15$ & 2.11 & 2.28 \\
\hline $\mathrm{Rb}$ & 0.43 & -0.37 & $0.17,-0.21$ & $2.12,2.72$ & $2.09,2.52$ \\
\hline $\mathrm{Cs}$ & 0.50 & -0.57 & $\cdots$ & $2.16,2.93$ & $2.35,2.50$ \\
\hline
\end{tabular}

${ }^{\text {a }}$ Reference 36 .

${ }^{\mathrm{b}}$ Reference 40 .

Since this dip has a width of about $20 k T$, it is unreasonable to suppose that the use of a step function is the cause.

Powell's analysis ${ }^{49}$ of Smith's results gives a value of $V_{110}=0.30 \mathrm{eV}$ for sodium which seems superficially to be in reasonable agreement with the dHvA value of $0.25 \mathrm{eV} .^{36}$ However, if $V_{110}^{\text {efp }}(T)$ is $0.25 \mathrm{eV}$ at near absolute zero then Fig. 2 shows that it will be about $0.19 \mathrm{eV}$ at $300^{\circ} \mathrm{K}$, a very important difference if the absorption varies as $\left(V_{110}\right)^{2}$.

There is no evidence in our calculations for the anomalous low-frequency peak observed by Mayer and Hietel in both sodium and potassium. Kopeliovich ${ }^{50}$ and Büttner and Gerlach ${ }^{51}$ attempt to explain this peak by phonon-assisted transitions to the $\mathrm{Bz}$ boundary (where the interband dipole matrix element is large). Miskovsky and Cutler have not resolved this possibility since their use of a free-electron energy spectrum and a wave function which is divergent at the zone boundary could not have enabled them to calculate correctly the contribution arising from phonon-assisted transitions which proceed by an intermediate state near the zone boundary. However, the phase space assoicated with these processes is small (if $V_{110}$ is small). The reason that Büttner and Gerlach get large quantitative estimates is that they use an electron-phonon "coupling constant" which does not allow for screening. Their coupling constant of $27 \mathrm{eV}$ is about 30 times larger than the correct energy $v(f)$, so their estimates are too high by about three orders of magnitude.

We find that the Drude absorption closely follows an $\omega^{-2}$ frequency dependence in sodium and potassium but we cannot rule out significant (i. e., experimentally observable) deviations for other metals. The HAA form factor ${ }^{15}$ gives excellent agreement with Smith's data in sodium at potassium. ${ }^{28}$ Finally, we conclude that, for the alkali metals at least, the common procedure of analyzing absorption curves as the sum of an $\omega^{-2}$ Drude term and a direct-interband term is invalid. A different situation may prevail in a polyvalent metal like aluminum where "parallel-band" absorption is so dominant. ${ }^{6}$

\section{ACKNOWLEDGMENTS}

I am very grateful to Professor D. Beaglehole for his guidance, and to Professor N. W. Ashcroft for many fruitful discussions. I wish to thank the Physics Department, Victoria University, for the prolonged use of computer facilities and the staff of the Laboratory of Atomic and Solid State Physics, Cornell University, for help in publishing this paper.

\footnotetext{
*Present address: Physics Department, Cornell University, Ithaca, N. Y. 14850.

${ }^{1}$ K. Sturm, J. Phys. F 2, 816 (1972).

${ }^{2}$ N. Miskovsky and P. Cutler, Phys. Rev. B $\underline{5}, 1265$ (1972).

${ }^{3}$ A. S. Karakashian and A. Bardasis, Phys. Rev. B $\underline{4}$, 404 (1971).

${ }^{4}$ J. E. Robinson and J. D. Dow, Phys. Rev. 171, 815 (1968); Phys. Rev. B $\underline{3}, 2818$ (1971).

${ }^{5}$ S. J. Nettel, Phys. Rev. 150, 421 (1966).

${ }^{6}$ N. W. Ashcroft and K. Sturm, Phys. Rev. B $\underline{3}, 1898$ (1971).

${ }^{7}$ C. J. Pethick, Phys. Rev. B $\underline{2}, 1789$ (1970).

${ }^{8}$ S. J. Nettel, J. Phys. Chem. Solids 29,2221 (1968).

${ }^{9}$ A. O. E. Animalu, Phys. Rev. 163, 557 (1967).

${ }^{10}$ B. A. Politzer, N. Miskovsky, and P. Cutler, Phys.
}

Letters 27A, 554 (1968).

${ }^{11}$ B. I. Bennett and S. H. Vosko, Phys. Rev. B $\underline{6}$, 2119 (1972).

${ }^{12}$ L. J. Sham, Proc. Phys. Soc. (London) 78, 895 (1961).

${ }^{13}$ M. J. Kelly (private communication).

${ }^{14} \mathrm{~J}$. G. Collins and J. M. Ziman, Proc. Roy. Soc. (London) 264, 60 (1961).

${ }^{15}$ W. A. Harrison, Pseudopotentials in the Theory of Metals (Benjamin, New York, 1966).

${ }^{16}$ N. W. Asheroft, J. Phys. C 1, 232 (1968).

${ }^{17} \mathrm{P}$. Sharma and S. Joshi, J. Chem. Phys. $\underline{39}, 2633$ (1963).

${ }^{18}$ D. L. Price, K. S. Singwi, and M. P. Tosi, Phys. Rev. B 2, 2983 (1970).

${ }^{19}$ P. N. Butcher, Proc. Phys. Soc. (London) A64, 765 
(1951).

${ }^{20}$ A. Ron, Phys. Rev. 131, 2041 (1963).

${ }^{21} \mathrm{E}$. Haga and T. Aisaka, J. Phys. Soc. Japan 22, 987 (1967).

${ }^{22} \mathrm{E}$. Wilson and S. Rice, Phys. Rev. 145, 55 (1966).

${ }^{23}$ L. J. Sham and J. M. Ziman, Solid State Phys. 15 , 221 (1963)

${ }^{24}$ J. S. Dugdale and D. Dugan, Proc. Roy. Soc. (London) 270,195 (1962) .

${ }^{25}$ W. Buyers and A. Cowley, Phys. Rev. 180, 755 (1969).

${ }^{26}$ R. H. Martinson, Phys. Rev. 178, 902 (1969).

${ }^{27}$ F. Ham, Phys. Rev. 128, 82 (1962).

${ }^{28}$ N. V. Smith, Phys. Rev. 183,634 (1969).

${ }^{29}$ N. V. Smith, Phys. Rev. B $\underline{2}, 2840$ (1970).

${ }^{30}$ R. Dawton, Proc. Roy. Soc. (London) 49, 294 (1937).

${ }^{31} \mathrm{G}$. V. Chester, Advan. Phys. 10, $357(\mathbf{1 9} 61)$.

${ }^{32}$ C. Y. Young, Phys. Rev. 183, 627 (1969); A. W.

Overhauser, ibid. 156, 844 (1967); M. Watabe and $\mathrm{H}$.

Yasuhara, Phys. Letters 34A, 295 (1971); R. N. Gurzhi

and M. I. Kaganov, Zh. Eksperim. i Teor. Fiz. $\underline{49}, 941$

(1945) [Sov. Phys. JETP 22, 654 (1966)].

${ }^{33} \mathrm{U}$. Whang, E. Arakava and I. Callcott, Phys. Rev. Letters 25, 646 (1970); J. G. Endriz and W. E. Spicer, Phys. Rev. B 4, 4144 (1971).

${ }^{34} \mathrm{H}$. Bennett, J. Bennett, E. Ashley, and R. Motyka, Phys. Rev. 165, 755 (1968).

${ }^{35}$ N. W. Ashcroft, Computational Methods in Band

Theory, edited by P. M. Marcus, J. F. Janak, and A. R. Williams (Plenum, New York, 1971), p. 368.

${ }^{36}$ M. J. Lee, Proc. Roy. Soc. (London) A295, 440

\author{
(1966). \\ ${ }^{37}$ M. Cohen and V. Heine, Solid State Phys. 24, 186 \\ (1970). \\ ${ }^{38}$ N. Wiser, Phys. Rev. 143, 393 (1966). \\ ${ }^{39}$ M. J. Lee and L. Falicov, Proc. Roy. Soc. (London) \\ A304, 319 (1968). \\ ${ }^{40}$ R. E. Palmer and S. E. Schnatterly, Phys. Rev. B \\ 4, 2329 (1971). \\ ${ }^{41} \mathrm{~S}$. Yamaguchi and T. Hanyu, J. Phys. Soc. Japan 31 , \\ 1431 (1971). \\ ${ }^{42} \mathrm{H}$. Mayer and $\mathrm{B}$. Hietel, in Optical Properties and \\ Electronic Structure of Metals and Alloys, edited by \\ Abelès (North-Holland, Amsterdam, 1966). \\ ${ }^{43} \mathrm{H}$. Mayer, D. L. Blanaru, and H. Steffen, Thin Solid \\ Films 5 , 389 (1970); H. Mayer and St. von Aufschnaiter, \\ Z. Physik 249, 400 (1972). \\ ${ }^{44} \mathrm{H}$. E. Ives and H. B. Briggs, J. Opt. Soc. Am. $\underline{27}$, \\ 395 (1937). \\ ${ }^{45}$ N. W. Ashcroft, Phys. Rev. 140, 935 (1965). \\ ${ }^{46} \mathrm{~J}$. Hodgson, in Ref. 40 . \\ ${ }^{47} \mathrm{~A}$. Mathewson and H. Myers, Report of Chalmers \\ University of Technology (Gothenburg, 1971) (unpublished); \\ Phil. Mag. 25, 853 (1972). \\ ${ }^{48}$ J. Callaway, .Phys. Rev. $\underline{124}, 1824$ (1961); 127,1913 \\ (1962). \\ ${ }^{49}$ C. J. Powell, Opt. Commun. 2 , 87 (1970). \\ ${ }^{50} \mathrm{~A}$. Kopeliovich, Zh. Eksperim. i Teor. Fiz. 58, 601 \\ (1970) [Sov. Phys. JETP 31, 323 (1970)]. \\ ${ }^{51} \mathrm{H}$. Büttner and E. Gerlach, J. Phys. Chem. Solids \\ $\underline{30}, 959(1969)$.
}

PHYSICAL REVIEW B

VOLUME 7, NUMBER 6

15 MARCH 1973

\title{
Pseudopotential Models for $\mathrm{Pb}$ and $\mathrm{Mg}$ : Convergence Properties
}

\author{
J. P. Van Dyke \\ Sandia Laboratories, Albuquerque, New Mexico 87115 \\ (Received 25 September 1972)
}

The convergence properties of Fermi-surface pseudopotential calculations are discussed. It is found for $\mathrm{Mg}$ and for $\mathrm{Pb}$ that previous models which use only a relatively small number of plane waves are inadequate, particularly when pressure derivatives of Fermi-surface cross sections are calculated. Convergence studies are presented for both materials. It is found for $\mathrm{Mg}$ that a local-pseudopotential model if converged can be satisfactory and thus that a nonlocal model is not required. For $\mathrm{Pb}$ it is found that several of the discrepancies between experimental results and the model of Anderson et al. can be attributed directly to nonconvergence of their model. A pseudopotential model for the $\mathrm{Pb}$ Fermi surface is presented which gives a quite satisfactory description of the de Haas-van Alphen results both at normal volume and under pressure.

\section{INTRODUCTION}

Parametrization of the Fermi surface of metals has become quite common. The parametrization may be done in a manner that is obviously mathematical ${ }^{1,2}$ such as a Kubic-harmonic expansion. Alternately, it may be considered in a more physical manner, ${ }^{3-6}$ for example, using a pseudopotential. The latter has certain pleasing features, particularly if one wishes to extend the model to com- pare with something beyond normal-volume de Haas-van Alphen (dHvA) information. 6, 7 The present work will be primarily concerned with the often neglected necessity for pseudopotential convergence if a pseudopotential model is to be a satisfactory physical model.

In Sec. II the pseudopotential method is reviewed. Section III considers $\mathrm{Mg}$, and we will see how the use of an unconverged pseudopotential led Kimball, Stark, and Mueller (KSM) $)^{5}$ to incorrectly conclude 